\title{
Genetic classification of ovarian carcinoma based on microsatellite analysis: Relationship to clinicopathological features and patient survival
}

\author{
ZHANG HUAN $^{1,4}$, KENTARO NAKAYAMA ${ }^{1}$, NAOMI NAKAYAMA ${ }^{1}$, MASAKO ISHIBASHI $^{1}$, \\ SHAMIMA YEASMIN ${ }^{1}$, ATSUKO KATAGIRI ${ }^{1}$, INDRI NURYANI PURWANA ${ }^{1}$, KOUJI IIDA ${ }^{1}$, \\ RIRUKE MARUYAMA $^{3}$, MANABU FUKUMOTO ${ }^{2}$ and KOHJI MIYAZAKI ${ }^{1}$
}

\author{
${ }^{1}$ Department of Obstetrics and Gynecology, Shimane University School of Medicine, Izumo; ${ }^{2}$ Department of Pathology \\ Institute of Development, Aging and Cancer, Tohoku University, Sendai; ${ }^{3}$ Department of Pathology \\ Shimane University School of Medicine, Izumo, Japan; ${ }^{4}$ Department of Gynecology \\ and Obstetrics, The Affiliated Hospital of Ningxia Medical College, P.R. China
}

Received June 29, 2007; Accepted September 28, 2007

\begin{abstract}
Ovarian carcinomas can progress through two pathways of genomic instability: chromosomal instability (CIN) and microsatellite instability (MSI). However, it is unknown whether these two mechanisms could be distinguished from each other in the molecular characteristics in ovarian carcinomas. We hypothesized that these two pathways are not always independent in ovarian carcinomas. We classified 51 ovarian carcinomas based on their MSI and CIN status using microsatellite analysis and assessed whether these carcinogenic pathways affect the clinicopathological features and patient survival. Of the 51 cases, $77.4 \%$ of the tumors were microsatellite stable (MSS), $5.9 \%$ were MSI-Low (MSI-L) whilst, $16.7 \%$ were MSI-High (MSI-H). Overall, $56.8 \%$ of the tumors had at least one loss of heterozygosity ( $\mathrm{LOH}$ ) event, i.e., $56.8 \%$ CIN. Notably, we identified a significant degree of overlap between the MSI and CIN pathways. Of the 34 tumors with $\mathrm{LOH}$ events (CIN), 5 (14.7\%) were MSI-H. In addition, of the 7 tumors that were MSI-H, 5 (71.4\%) had one or more LOH events (CIN). We also identified a group of $29.4 \%$ of all tumors that did not demonstrate any evidence of either of the two
\end{abstract}

Correspondence to: Dr Kentaro Nakayama, Department of Obstetrics and Gynecology, Shimane University School of Medicine, Enyacho 89-1, Izumo 693-8501, Japan

E-mail:kn88@med.shimane-u.ac.jp

Abbreviations: LOH, loss of heterozygosity; CIN, chromosomal instability; MSI, microsatellite instability; MSS, microsatellite stable; MMR, mismatch repair

Key words: ovarian carcinoma, loss of heterozygosity, microsatellite instability, genomic instability, prognosis pathways of genomic instability as they were MSS/MSI-L with no evidence of LOH events (CIN negative). Furthermore, patients with CIN with MSS/MSI-L have a significantly shorter overall survival compared to those in other genetic categories $(\mathrm{P}=0.019)$. Cox regression analysis revealed that tumors with CIN with MSS/MSI-L exhibit a poor prognostic outcome after adjustment for FIGO stage and grade. These findings suggest that some ovarian carcinomas have a significant degree of overlap between the two pathways of genomic instability and that the genetic classification using microsatellite markers may represent a potential new biomarker of risk prediction in ovarian carcinoma.

\section{Introduction}

Ovarian carcinoma is the most lethal gynecological malignancy at present (1). Women with organ-confined tumors have an excellent prognosis, whereas the overall survival of the majority of ovarian carcinoma patients with advanced disease is $<30 \%(2,3)$. Despite the development of new therapeutic approaches, these survival statistics have remained largely unchanged for many years. Clearly, there is a need for a better understanding of the molecular pathogenesis of ovarian carcinoma so that new drug targets or biomarkers that facilitate risk prediction can be identified.

Ovarian carcinogenesis is characterized by the successive accumulation of mutations in genes controlling epithelial cell growth and differentiation. The term 'genomic instability' describes conditions in which widespread loss of DNA integrity is perpetuated. The development of genomic instability is an important event in the multistep progression of ovarian carcinogenesis. Two apparently independent pathways of genomic instability have been identified $(4,5)$. The first, and more common pathway is characterized by the sequential inactivation of tumor-suppressor genes, such as p53 (chromosome 17p), BRCAl (chromosome 17q) and FHIT (chromosome 3p). Tumors generated through this 
'suppressor' pathway display chromosal instability (CIN) with frequent cytogenetic abnormalities and allelic losses $(6,7)$. The precise mechanism driving the process of chromosomal instability is not completely understood. A second pathway is characteristic of tumors from patients with HNPCC, an autosomal-dominat condition that accounts for $2-3 \%$ of all colorectal carcinomas. The hallmark of this alternative 'mutator' pathway is widespread microsatellite instability (MSI), which is characterized by the accumulation of somatic alterations in the length of simple, repeated nucleotide sequences called 'microsatellites'.

Microsatellite instability (MSI) is also seen in a significant proportion of extracolonic tumors including breast, endometrial (8), gastric (9), and ovarian carcinoma (10-12). MSI-H has also been identified in $3-10 \%$ of sporadic solid carcinomas (13). In these cases, mutations of $h M L H 1$ and $h M S H 2$ are rarely found. Some studies indicate that $h M L H 1$ inactivation by promoter hypermethylation also produces the MSI-H phenotype in sporadic solid carcinomas and is responsible for most, if not all, of the sporadic solid carcinomas exhibiting MSI-H (14-16). MSI-H colorectal carcinomas do not exhibit gross cytogenetic abnormalities; and they are not generally aneuploid $(17,18)$. Furthermore, patients with MSI-H tumors have a more favorable survival compared to patients with MSI-L/MSS solid carcinomas $(13,19,20)$.

Although these two mechanisms of genomic instability can be distinguished from one another by their molecular characteristics, current evidence suggests that there might be some degree of overlap. Recently, Goel et al reported that there is a significant degree of overlap between the MSI and CIN pathways in colorectal carcinomas (21). To date, no systematic study has determined the extent of overlap between the MSI and CIN pathways in ovarian carcinomas. The present study was therefore pursued in order to classify ovarian carcinomas based on their MSI and CIN status. We hypothesized that some tumors would exhibit a significant degree of overlap between these two mechanisms, whilst a proportion of tumors might not show any evidence for involvement of either mutational pathways. In addition, we assessed whether evidence of these carcinogenic pathways was related to clinicopathological features and patient survival.

\section{Materials and methods}

Patients and tumor samples. Fifty-one ovarian carcinomas and their adjacent non-neoplastic tissues were obtained from archival pathological specimens from the Shimane University Hospital in Japan. Written informed consent for the analysis in this study was obtained for each individual case. Acquisition of tissue specimens was approved by the Institutional Review Board at Shimane University. Diagnostic verification, tumor subtyping and grading were performed independently by two certified pathologists (R.M. and M.F.). Ovarian carcinomas were diagnosed on the basis of conventional histopathological criteria (22), using the grading criteria recommended by the International Federation of Gynecology and Obstetrics.

The series of tumor tissues included in the study consisted of 20 serous, 17 mucinous, 11 endometrioid and 3 clear cell ovarian carcinomas. The 51 tumors included 24 welldifferentiated (grade 1), 14 moderately differentiated (grade 2) and 13 poorly differentiated (grade 3 ) ovarian carcinomas. There were 22 stage I patients, 5 stage II patients, 19 stage III patients and 5 stage IV patients in this study.

Patients had no other malignancies and had performance status of grade 0 or 1 . All patients were primarily treated with cytoreductive surgery and postoperative chemotherapy, which consisted of 6-12 courses of a paclitaxel-carboplatin regimen (TC; paclitaxel $175 \mathrm{mg} / \mathrm{m}^{2}$, CBDCA AUC5). Chemotherapy response was assessed by second-look surgery or by clinical and/or radiographic evaluation according to the WHO criteria. Patients with incomplete responses to induction chemotherapy or with recurrent tumors, were treated with a variety of second-line chemotherapy regimens. Follow-up for all patients included in the survival analysis was updated June 25, 2003 (median follow-up time was 80 months; range, 50-120 months). At that time, 27 patients had died of ovarian carcinoma.

DNA extraction using microdissection and PCR amplification. Paraffin-embedded tissues were sectioned at a thickness of $5 \mu \mathrm{m}$ and stained with hematoxylin and eosin. The cancerous and non-neoplastic portions were collected separately with a 29-gauge needle using an MK1 micromanipulator (Singer Instruments, Roadwater, UK) under a dissecting microscope. The dissected tissue was collected in an Eppendorf tube and incubated overnight at $58^{\circ} \mathrm{C}$ in a digestion mixture $(0.01 \mathrm{M}$ $\mathrm{NaCl} ; 0.5 \mathrm{M}$ Tris- $\mathrm{HCl}, \mathrm{pH} 8.0 ; 20$ mM EDTA; $0.05 \%$ Tween- $20^{\circledR} ; 0.1 \mathrm{mg} / \mathrm{ml}$ proteinase $\left.\mathrm{K}\right)$. The samples were then heated to $95^{\circ} \mathrm{C}$ for $10 \mathrm{~min}$ to inactivate the proteinase $\mathrm{K}$ activity. After digestion, DNA was extracted with phenol/ chloroform treatment and ethanol precipitation. PCR reactions were performed in a total volume of $10 \mu \mathrm{l}$ containing 25-50 ng of DNA, dNTPs at a final concentration of $20 \mu \mathrm{M}, 0.4 \mu \mathrm{M}$ of each primer, and 0.25 units of Ex-Taq DNA polymerase (Takara Shuzo, Shiga, Japan) or Platinum Taq DNA polymerase (Gibco Brl, Rockville, MD). After the mixture was heated for $10 \mathrm{~min}$ at $94^{\circ} \mathrm{C}, \mathrm{PCR}$ was performed for 45 cycles at $94^{\circ} \mathrm{C}$, at the appropriate annealing temperature and at $72^{\circ} \mathrm{C}$ for $1 \mathrm{~min}$ each, followed by $72^{\circ} \mathrm{C}$ for $10 \mathrm{~min}$. After denaturation of the PCR products at $94^{\circ} \mathrm{C}$ for 2 min, samples were subjected to electrophoresis using Performance optimized polymer 4 in a 310 Genetic analyzer (Applied Biosystems, Foster, CA). LOH analysis was performed by Gene Scan version 2.1.

Microsatellite markers and MSI analysis. Allelic imbalances were measured by performing MSI on all matched normal and tumor tissues by PCR amplification. A panel of eight microsatellite markers with dye-labeled primers, comprising of 7 dinucleotide repeats (D2S123, D5S346, D8S87, D13S153, D13S175, D18S55, D20S100) and, 1 mononucleotide repeat (BAT25) were used to determine tumor MSI status. These markers included the recommended markers for the detection of MSI proposed at the National Cancer Institute collaborative meeting on MSI in colorectal carcinoma (23). Loci were scored according to the published guidelines. Changes in the electrophoretic mobility of DNA amplified by PCR were used to assess the MSI. We defined tumors as exhibiting MSI if they had at least 1 locus with MSI among cases in which PCR was successful on more than 
Table I. Association of microsatellite genotypes with clinicopathological features.

\begin{tabular}{|c|c|c|c|c|c|}
\hline & $\begin{array}{l}\mathrm{CIN} \\
\mathrm{n}=34\end{array}$ & $\begin{array}{c}\text { CIN negative } \\
\mathrm{n}=17\end{array}$ & $\begin{array}{c}\text { MSI-H } \\
\mathrm{n}=7\end{array}$ & $\begin{array}{c}\text { MSI-L/MS } \\
\mathrm{n}=47\end{array}$ & P-value \\
\hline \multicolumn{6}{|l|}{ Histological type } \\
\hline Serous $(n=20)$ & 13 & 10 & 1 & 22 & $0.07^{\mathrm{a}}$ \\
\hline Mucinous $(n=17)$ & 12 & 4 & 2 & 14 & \\
\hline Endometrioid $(\mathrm{n}=11)$ & 6 & 3 & 4 & 5 & \\
\hline Clear $(n=3)$ & 3 & 0 & 0 & 3 & \\
\hline \multicolumn{6}{|l|}{ Grade } \\
\hline Well $(n=24)$ & 12 & 11 & 6 & 17 & $0.02^{\mathrm{b}}$ \\
\hline Moderate $(n=14)$ & 11 & 2 & 0 & 13 & \\
\hline Poor $(n=13)$ & 11 & 4 & 1 & 14 & \\
\hline \multicolumn{6}{|l|}{ FIGO stage/TNM stage } \\
\hline $\mathrm{I} / \mathrm{Tl}(\mathrm{n}=22)$ & 12 & 9 & 5 & 16 & $0.04^{\mathrm{c}}$ \\
\hline II/T2 (n=5) & 2 & 3 & 1 & 4 & $0.04^{\mathrm{d}}$ \\
\hline III/T3 (n=19) & 17 & 4 & 1 & 20 & \\
\hline IV/T4 $(n=5)$ & 3 & 1 & 0 & 4 & \\
\hline
\end{tabular}

${ }^{a}$ MSI-H tumors tended to be more frequent in endometrioid type tumors than in serous type tumors. ${ }^{b} \mathrm{MSI}-\mathrm{H}$ tumors were significantly more frequent in well differentiated carcinomas than in moderately/poorly differentiated carcinomas. ${ }^{c} \mathrm{MSI}-\mathrm{H}$ tumors were significantly more frequent in stage I, II tumors than in stage III, IV tumors. ${ }^{\mathrm{d}} \mathrm{IN}$ tumors were significantly more frequent in stage III, IV tumors than in stage I, II tumors.

five loci. Among tumors exhibiting MSI, cases with $>30 \%$ of loci showing MSI were defined as MSI-H and the remaining cases were designated MSI-L. Tumors not demonstrating allelic shifts were termed as MSS (Fig. 1).

Microsatellite markers and LOH analysis. Eight sets of polymorphic microsatellite sequences that are tightly linked to known tumor suppressor genes and markers reported to be associated with ovarian carcinoma were used to identify significant allelic losses in the carcinoma specimens. DNA was amplified by PCR using dye-labeled primers at microsatellite loci linked to the FHIT locus on 3p21.2-14.2 (D3S1306), p53 locus on 17p13 (TP53), BRCA1 locus on 17q21 (D17S579), 6q25 (D6S473), 7q31 (D7S523), 9q31-33 (D9S59), 11p15.5-15.3 (D11S988) and Xq11-12 (AR). LOH was quantified by calculating the $\mathrm{LOH}$ index. This was defined as the allele ratio in normal tissue divided by the allele ratio in tumor tissue. The allele ratio was calculated as the peak height of the smaller allele divided by the peak height of the larger allele. If the $\mathrm{LOH}$ index was $<0.5$ or $>2.0$, we defined the case as LOH. The LOH frequency of each locus was represented by the ratio of the number of cases with $\mathrm{LOH}$ to the total number of informative cases.

Statistical analysis. Overall survival time was measured in months from the time of surgery to the reported death. Survival curves were determined using the Kaplan-Meier method and differences in survival between subgroups were compared with the log-rank test. P-values $<0.05$ were considered significant. All reported P-values are two-tailed.

\section{Results}

Microsatellite typing. Informative results were obtained for all tumor specimens. Of the 51 cases, $41(77.4 \%)$ were MSS, 3 (5.9\%) were MSI-L, and 7 (16.7\%) were MSI-H (Table I). MSI-L tumors were categorized along with MSS tumors for all statistical purposes in this study. MSI-H tumors correlated with both histological subtype and tumor grade. MSI-H tumors tended to be more frequent in endometrioid type tumors than in serous type tumors $(\mathrm{P}=0.07)$ (Table I). In addition, MSI-H tumors were significantly more frequent in well-differentiated carcinomas than in moderately/poorly differentiated carcinomas $(\mathrm{P}=0.02)$. MSI-H tumors were significantly more frequent in stage I, II tumors than in stage III, IV tumors $(\mathrm{P}=0.04)$.

LOH study. We identified 34 tumors (66.7\%) with $\mathrm{LOH}$ at one or more of the eight loci studied. Overall, the frequency of $\mathrm{LOH}$ in tumors with any LOH event was most common in $17 \mathrm{p} 13$ (TP53) (46.3\%), followed by 11p15.5-15.3 (D11S988) (38.7\%), 3p21.2-14.2 (D3S1306) (34.8\%), 9q31-33 (D9S59) (30.0\%), 7q31 (D7S523) (29.4\%), 6q25 (D6S473) (21.0\%), Xq11-12 (AR) (15.0\%) and 17q21 (D17S579) (13.2\%). Based on the number of affected $\mathrm{LOH}$, the LOH genotype was divided into 2 groups. Tumors with LOH in at least one of the eight markers were classified as exhibiting chromosomal instability (CIN) whilst tumors with no evidence of $\mathrm{LOH}$ were classified as CIN negative. Of the 53 cases, 34 (66.7\%) were CIN positive and $17(33.3 \%)$ were CIN negative (Table I). CIN tumors were significantly more frequent in stage III, IV tumors than in stage I, II tumors $(\mathrm{P}=0.04)$. 

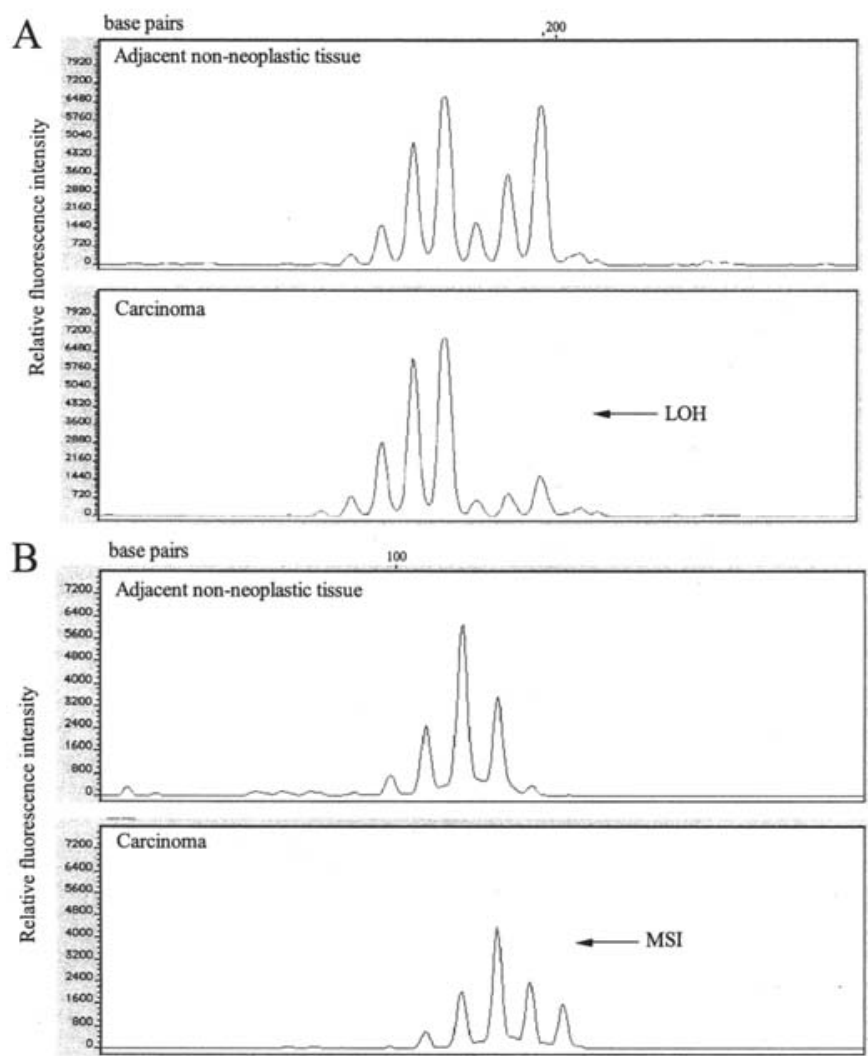

Figure 1. Representative example of the loss of heterozygosity (LOH) and microsatellite instability (MSI).

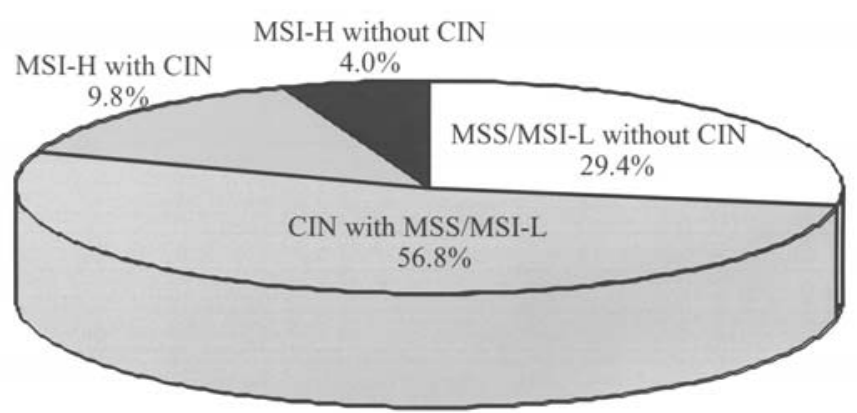

Figure 2. Exclusiveness and overlap among subsets of genomic instability. Summary of genomic instability patterns for 51 ovarian carcinomas.

Overlap of different pathways of genomic instability. We then investigated the degree of overlap between tumors with CIN and those with MSI. Of the 34 tumors with evidence of CIN, $85.3 \%$ were MSS/MSI-L and $14.7 \%$ were MSI-H (Table I). Of the $44 \mathrm{MSS} / \mathrm{MSI}-\mathrm{L}$ tumors, $65.9 \%$ also contained a LOH event at one or more of the loci tested (CIN), as did $71.4 \%$ of the MSI-H tumors. The proportions of CIN positive tumors did not differ according to MSI status. We also examined the distribution of tumors that exhibited the following combinations: CIN and MSS/MSI-L, CIN and MSI-H, MSI-H without CIN, and MSS/MSI-L without CIN (Fig. 2). We found that $9.8 \%$ of all tumors exhibited both MSI-H and CIN whilst, $4.0 \%$ were negative for both MSI-H and CIN. In addition, $56.8 \%$ of the MSS/MSI-L tumors were CIN negative. It is
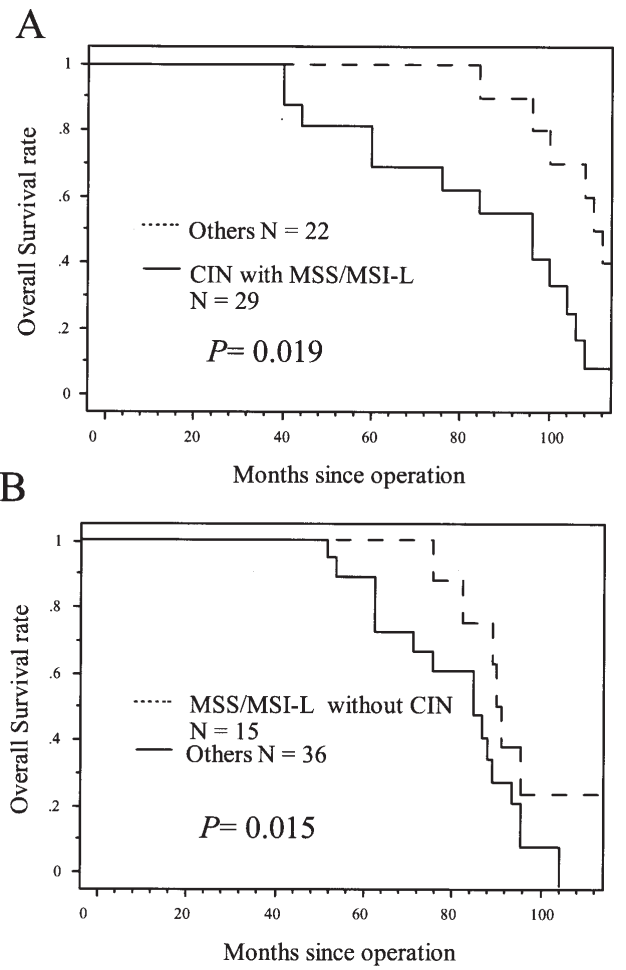

Figure 3. Kaplan-Meier curves of 51 ovarian carcinomas according to the status of genomic instability. (A) Patients with CIN with MSS/MSI-L have a significantly shorter overall survival compared with other genetic categories $(\mathrm{P}=0.019)$. (B) Patients with MSS/MSI-L without CIN tended to have a favorable overall survival compared with other genetic categories $(\mathrm{P}=0.151)$.

noteworthy that we identified a group comprising of $29.4 \%$ of all tumors examined that were MSS/MSI-L and CIN negative and therefore did not demonstrate signs of either of the two pathways of genomic instability (Fig. 2).

Correlation between status of genomic instability and clinicopathological features. Comparisons of the clinical and pathological variables of these four tumor categories are provided in Table II. There was no significant difference in the relationship between the status of genomic instability and histological subtype. With respect to the histological grade, a significantly greater fraction of tumors with CIN with MSS/MSI-L were observed in well-differentiated carcinomas compared with tumors exhibiting MSS/MSI-L without CIN $(\mathrm{P}=0.034,0.036$; well vs moderate, well vs moderate + poor $)$. Patients were grouped as early stage (stage I and II) and advanced stage (stage III and IV) carcinomas. We found that 19 out of 29 CIN positive tumors with MSS/MSI-L types $(65.5 \%)$ were advanced stage. On the other hand, 10 out of 15 CIN negative tumors with MSS/MSI-L types (66.7\%), 4 out of 5 CIN positive tumors with MSI-H types (80.0\%) and 2 out of 2 CIN negative tumors with MSI-H types (100\%) were more common in the early stage. CIN positive tumors with MSS/MSI-L types exhibited a significantly more advanced stage compared with CIN negative tumors with MSS/MSI-L types $(\mathrm{P}=0.042)$.

Analysis of survival rate. To verify whether a specific genomic instability exhibits a preferential association with more or less 
Table II. Correlation between status of genomic instability and clinicopathological features.

\begin{tabular}{|c|c|c|c|c|c|c|}
\hline & $\begin{array}{c}\text { CIN with } \\
\text { MSS/MSI-L }(n=29)\end{array}$ & $\begin{array}{l}\text { MSI-H without } \\
\text { CIN }(\mathrm{n}=2)\end{array}$ & $\begin{array}{l}\text { MSI-H with } \\
\text { CIN }(n=5)\end{array}$ & $\begin{array}{l}\text { MSS/MSI-L without } \\
\text { CIN }(\mathrm{n}=15)\end{array}$ & $\begin{array}{l}\text { Total } \\
(\mathrm{n}=51)\end{array}$ & P-value \\
\hline \multicolumn{7}{|l|}{ Histological type } \\
\hline Serous $(n=20)$ & 13 & 1 & 0 & 9 & 20 & \\
\hline Mucinous $(n=17)$ & 10 & 0 & 2 & 4 & 17 & \\
\hline Endometrioid (n=11) & 3 & 1 & 3 & 2 & 11 & \\
\hline Clear $(n=3)$ & 3 & 0 & 0 & 0 & 3 & \\
\hline \multicolumn{7}{|l|}{ Grade } \\
\hline Well $(n=24)$ & 8 & 2 & 4 & 9 & 24 & $0.034^{\mathrm{a}}$ \\
\hline Moderate $(n=14)$ & 11 & 0 & 0 & 2 & 14 & $0.036^{\mathrm{b}}$ \\
\hline Poor $(n=13)$ & 10 & 0 & 1 & 4 & 13 & \\
\hline \multicolumn{7}{|l|}{ FIGO stage/TNM stage } \\
\hline $\mathrm{I} / \mathrm{T} 1(\mathrm{n}=22)$ & 9 & 2 & 3 & 7 & 22 & $0.042^{\mathrm{c}}$ \\
\hline II/T2 (n=5) & 1 & 0 & 1 & 3 & 5 & \\
\hline III/T3 (n=19) & 16 & 0 & 1 & 4 & 19 & \\
\hline IV/T4 (n=5) & 3 & 0 & 0 & 1 & 5 & \\
\hline
\end{tabular}

${ }^{\mathrm{a}, \mathrm{b}} \mathrm{A}$ significantly greater fraction of tumors with CIN with MSS/MSI-L were observed in well-differentiated carcinomas compared with tumors exhibiting MSS/MSI-L without CIN (well vs moderate, well vs moderate + poor). ${ }^{\circ} \mathrm{CIN}$ with MSS/MSI-L tumors exhibited a significantly more advanced stage compared with MSS/MSI-L without CIN tumors.

Table III. Multivariate analysis of overall survival in ovarian carcinoma patients.

\begin{tabular}{|c|c|c|c|c|}
\hline \multirow[b]{2}{*}{ Variables } & \multirow{2}{*}{$\frac{\text { Univariate }}{\text { P-value }}$} & \multicolumn{3}{|c|}{ Multivariate } \\
\hline & & Hazard ratio & $95 \% \mathrm{CI}$ & P-value \\
\hline \multicolumn{5}{|l|}{ FIGO stage } \\
\hline I, II (n=27) vs III, IV (n=24) & 0.0003 & 7 & $1.2-38.7$ & 0.027 \\
\hline \multicolumn{5}{|l|}{ Histological grade } \\
\hline Poorly differentiated $(n=13)$ vs Others $(n=38)$ & 0.0126 & 1.6 & $0.3-7.5$ & 0.545 \\
\hline \multicolumn{5}{|l|}{ Status of genomic instability } \\
\hline CIN with MSS/MSI-L $(n=29)$ vs Others $(n=22)$ & 0.02 & 2.5 & $1.0-6.0$ & 0.042 \\
\hline
\end{tabular}

favorable clinical course, the overall survival of patients with all genetic categories were analyzed using the Kaplan-Meier method. We analyzed the overall survival in CIN-positive tumors with CIN-negative tumors and MSI-H tumors with MSS/MSI-L tumors. The patients with CIN-positive tumors tended to have a shorter overall survival compared to those with CIN-negative tumors ( $\mathrm{P}=0.099)$. In contrast, MSI-H had no influence on patient survival $(\mathrm{P}=0.421)$. Then, we compared overall survival of the four categories described above (i.e., CIN with MSS/MSI-L, MSI-H without CIN, MSI-H with CIN and MSS/MSI-L without CIN). Patients with CIN with MSS/ MSI-L had a significantly shorter overall survival compared with other genetic categories $(\mathrm{P}=0.019)$ (Fig. 3A). However, patients with MSS/MSI-L without CIN tended to have a more favorable overall survival compared with other genetic categories $(\mathrm{P}=0.151)$ (Fig. 3B). No statistically significant difference was evident in the survival curves of patients with the other two genetic classifications $(\mathrm{P}=0.644$ and 0.838 , respectively). To determine whether CIN-positive tumors with
MSS/MSI-L were a prognostic marker independent of FIGO stage or histological grade established prognostic markers, we conducted an overall survival analysis using the Cox proportional hazards model (Table III). FIGO stage (hazard ratio: $7.0,95 \% \mathrm{CI}: 1.2-38.7, \mathrm{P}=0.027)$ and $\mathrm{CIN}$ with MSS/MSI-L (hazard ratio: 2.5, 95\% CI: $1.0-6.0, \mathrm{P}=0.042$ ) were independent prognostic factors in patients with ovarian carcinoma (Table III).

\section{Discussion}

Our current understanding of solid tumor carcinogenesis, including ovarian carcinoma suggests that at least two mechanisms are capable of producing the mutations required for a cell to demonstrate a malignant phenotype. These mechanisms include CIN, characterized in tumor DNA by the presence of multiple $\mathrm{LOH}$ events, and loss of MMR function that is defined by high levels of MSI. In the current study, we examined 51 cases of ovarian carcinoma to determine whether 
these two mechanisms of tumorigenesis operated independently or whether there exists a significant degree of overlap.

We defined tumors as MSI if they had at least 1 locus with MSI among cases in which PCR was successful at more than 5 loci. Among MSI tumors, cases with $>30 \%$ of loci showing MSI were defined as MSI-H and the remaining cases were designated MSI-L. Some studies suggest that the molecular profiles of MSS and MSI-L tumors are indistinguishable $(24,25)$ and we therefore, categorized MSI-L tumors with MSS tumors for all statistical purposes. To investigate allelic losses by $\mathrm{LOH}$, we used eight polymorphic markers mapped closely to key tumor suppressor genes that are believed to be lost during ovarian carcinogenesis (26-32). Detection of $\mathrm{LOH}$ in at least one of these sites was taken as evidence of loss of tumor suppressor activity by CIN. We accept that it is possible and even likely that tumors may well be reclassified by the addition of markers outside the usual deletion sites. However, without a single LOH event at the eight sites examined, it is highly unlikely that a tumor would exhibit widespread $\mathrm{LOH}$ that is characteristic of tumors arising in the setting of CIN. With these data, we classified the tumors as belonging to one of four genotypes: CIN with MSS/MSI-L; CIN with MSI-H; MSI-H without CIN and MSS/MSI-L without CIN.

MSI-H without CIN was found in $4 \%$ of the tumors analyzed. MSI-H is caused by a defect in DNA MMR capability which is most commonly caused by hypermethylation of the hMLH1 promoter (33). Carcinomas associated with MMR defect are typically diploid, although comparative genomic hybridization shows that these tumors may also demonstrate amplifications and deletions of single alleles or chromosomes (34). In agreement with this, $9.8 \%$ of the tumors in our study exhibited both MSI-H and CIN.

CIN, as evidenced by allelic loss at one or more of the eight markers tested, was observed in $66.7 \%$ of the tumors examined. Tumors that exhibited LOH without MSI-H comprised $56.8 \%$ of the tumors. The presumed course of tumor progression in this subset involves accumulated allelic losses at the tumor suppressor loci (4). The processes responsible for CIN are unknown. Many mechanistic explanations have been offered for CIN, but none have been shown to be sufficient to account for this process in solid tumor carcinogenesis $(7,35,36)$. It is likely that multiple causes of CIN will be discovered through the study of these and other events in early carcinogenesis.

The most intriguing subgroup identified in this study was the $29.4 \%$ of ovarian carcinomas that lacked any evidence of either CIN or MSI. The molecular events leading to the development of carcinomas in this subgroup with no signs of genomic instability are unknown. One reasonable possibility is the transcriptional silencing of growth and differentiation genes by epigenetic modification. Aberrant promoter methylation, leading to loss of the tumor suppressor function, has been observed in a variety of cancers (37). Epigenetic modification of tumor suppressor genes is characteristically age dependent (38) and there are several reports in the literature regarding tumor-specific methylation patterns (39). We are in the process of characterizing the MSS/MSI-L without CIN subset using methylation-specific PCR.

We then assessed whether these carcinogenic pathways as discussed above, affect the clinical prognosis. It is well accepted that clinical stage is the most reliable guide to a prognosis. A biomarker might provide additional prognostic information if it were directly linked to a mechanism driving invasion and metastasis. Many investigators reported that MSI solid carcinomas are associated with an improved prognosis $(13,19,20)$, but the reasons are unclear $(40)$. In the current study, MSI ovarian carcinomas had no influence on patient survival $(\mathrm{P}=0.421)$. The emergence of carcinoma cells that are resistant to cisplatin and carboplatin is a major clinical problem and often leads to tumor recurrence (41). Ovarian carcinoma cell lines that develop resistance to cisplatin have been found to acquire an MSI-H phenotype (42). Therefore, MSI-H ovarian carcinomas might not be associated with an improved prognosis in this study because all ovarian carcinoma patients in this study received cisplatin based chemotherapy.

One of the most statistically significant findings in our study was that patients with CIN with MSS/MSI-L ovarian carcinomas had a poor prognosis. CIN can be interpreted as indicating a high level of deleted chromosomal regions, aberrant mitotic recombinations, or nondisjunctional chromosomal loss (43). In addition, it is also possible that some of the allelic imbalances we observed were due to the amplification of certain chromosomal regions. While genetic instability has generally been considered to be a characteristic feature of malignancy, it is still a matter of debate whether this instability is causally related to carcinogenesis or merely the result of other cellular events. Recent studies of colon carcinoma cells exhibiting CIN indicated that the CIN phenotype appeared to be a causative factor for tumor development, and may result from defects in proteins participating in mitotic checkpoint control (6). The striking association between tumors with CIN with MSS/MSI-L and a poor prognosis, as observed in our study, may be explained by the possibility that CIN reflects an aggressive tumor phenotype. Our findings appear to fit the concept that specific types of genetic instability exist, and that this instability has a role in driving tumor development and progression (44).

In summary, we reported that some ovarian carcinomas have a significant degree of overlap between the two pathways of genomic instability: CIN and MSI. The results of our study suggest that microsatellite typing is a useful tool for the construction of a comprehensive genetic classification that aims to predict the diverse clinical prognosis. A genetic classification using microsatellite markers may represent a potential new biomarker of risk prediction in ovarian carcinoma.

\section{References}

1. Wingo PA, Tong T and Bolden S: Cancer statistics, 1995. CA Cancer J Clin 45: 8-30, 1995.

2. Landis SH, Murray T, Bolden S and Wingo PA: Cancer statistics, 1999. CA Cancer J Clin 49: 8-31, 1999.

3. Holschneider CH and Berek JS: Ovarian cancer: epidemiology, biology, and prognostic factors. Semin Surg Oncol 19: 3-10, 2000 .

4. Kinzler KW and Vogelstein B: Lessons from hereditary colorectal cancer. Cell 87: 159-170, 1996.

5. Toft NJ and Arends MJ: DNA mismatch repair and colorectal cancer. J Pathol 185: 123-129, 1998.

6. Lengauer C, Kinzler KW and Vogelstein B: Genetic instabilities in human cancers. Nature 396: 643-649, 1998. 
7. Cahill DP, Lengauer C, Yu J, Riggins GJ, Willson JK, Markowitz SD, et al: Mutations of mitotic checkpoint genes in human cancers. Nature 392: 300-303, 1998.

8. Gurin CC, Federici MG, Kang L and Boyd J: Causes and consequences of microsatellite instability in endometrial carcinoma. Cancer Res 59: 462-426, 1999.

9. Leung WK, Kim JJ, Kim JG, Graham DY and Sepulveda AR: Microsatellite instability in gastric intestinal metaplasia in patients with and without gastric cancer. Am J Pathol 156: 537-543, 2000.

10. Sood AK and Buller RE: Genomic instability in ovarian cancer: a reassessment using an arbitrarily primed polymerase chain reaction. Oncogene 13: 2499-2504, 1996.

11. Hickey KP, Boyle KP, Jepps HM, Andrew AC, Buxton EJ and Burns PA: Molecular detection of tumour DNA in serum and peritoneal fluid from ovarian cancer patients. Br J Cancer 80: 1803-1808, 1999

12. Sood AK, Holmes R, Hendrix MJ and Buller RE: Application of the National Cancer Institute international criteria for determination of microsatellite instability in ovarian cancer. Cancer Res 61: 4371-4374, 2001.

13. Lawes DA, SenGupta S and Boulos PB: The clinical importance and prognostic implications of microsatellite instability in sporadic cancer. Eur J Surg Oncol 29: 201-212, 2003.

14. Cunningham JM, Christensen ER, Tester DJ, Kim CY, Roche PC Burgart LJ, et al: Hypermethylation of the hMLH1 promoter in colon cancer with microsatellite instability. Cancer Res 58: 3455-3460, 1998

15. Kuismanen SA, Holmberg MT, Salovaara R, Schweizer P, Aaltonen LA, de La Chapelle A, et al: Epigenetic phenotypes distinguish microsatellite-stable and -unstable colorectal cancers. Proc Natl Acad Sci USA 96: 12661-12666, 1999.

16. Murata H, Khattar NH, Kang Y, Gu L and Li GM: Genetic and epigenetic modification of mismatch repair genes hMSH2 and hMLH1 in sporadic breast cancer with microsatellite instability. Oncogene 21: 5696-5703, 2002.

17. Olschwang S, Hamelin R, Laurent-Puig P, Thuille B, De Rycke Y, Li YJ, et al: Alternative genetic pathways in colorectal carcinogenesis. Proc Natl Acad Sci USA 94: 12122-12127, 1997.

18. Eshleman JR, Casey G, Kochera ME, Sedwick WD, Swinler SE, Veigl ML, et al: Chromosome number and structure both are markedly stable in RER colorectal cancers and are not destabilized by mutation of p53. Oncogene 17: 719-725, 1998 .

19. Bubb VJ, Curtis LJ, Cunningham C, Dunlop MG, Carothers AD Morris RG, et al: Microsatellite instability and the role of hMSH2 in sporadic colorectalcancer. Oncogene 12: 2641-2649, 1996.

20. Watson P, Lin KM, Rodriguez-Bigas MA, Smyrk T, Lemon S, Shashidharan M, et al: Colorectal carcinoma survival among hereditary nonpolyposis colorectal carcinoma family members. Cancer 83: 259-266, 1998.

21. Goel A, Arnold CN, Niedzwiecki D, Chang DK, Ricciardiello L, Carethers JM, et al: Characterization of sporadic colon cancer by patterns of genomic instability. Cancer Res 63: 1608-1614, 2003.

22. Serov SF, Scully RE and Sobin LH: Histopathological typing of ovarian tumors: International classification of tumors. Vol. 9, WHO, Geneva, 1973.

23. Boland CR, Thibodeau SN, Hamilton SR, Sidransky D, Eshleman JR, Burt RW, et al: A National Cancer Institute Workshop on Microsatellite Instability for cancer detection and familial predisposition: development of international criteria for the determination of microsatellite instability in colorectal cancer. Cancer Res 58: 5248-5257, 1998.

24. Kambara T, Matsubara N, Nakagawa H, Notohara K, Nagasaka T, Yoshino T, et al: High frequency of low-level microsatellite instability in early colorectal cancer. Cancer Res 61: 7743-7746, 2001

25. Halford S, Sasieni P, Rowan A, Wasan H, Bodmer W, Talbot I, et al: Low-level microsatellite instability occurs in most colorectal cancers and is a nonrandomly distributed quantitative trait. Cancer Res 62: 53-57, 2002.
26. Fullwood P, Marchini S, Rader JS, Martinez A, Macartney D, Broggini M, et al: Detailed genetic and physical mapping of tumor suppressor loci on chromosome $3 \mathrm{p}$ in ovarian cancer. Cancer Res 59: 4662-4667, 1999.

27. Colitti CV, Rodabaugh KJ, Welch WR, Berkowitz RS and Mok SC: A novel $4 \mathrm{cM}$ minimal deletion unit on chromosome 6q25.1-q25.2 associated with high grade invasive epithelial ovarian carcinomas. Oncogene 16: 555-559, 1998.

28. Koike M, Takeuchi S, Yokota J, Park S, Hatta Y, Miller CW, et al: Frequent loss of heterozygosity in the region of the D7S523 locus in advanced ovarian cancer. Genes Chromosomes Cancer 19: $1-5,1997$

29. Devlin J, Elder PA, Gabra H, Steel CM and Knowles MA: High frequency of chromosome 9 deletion in ovarian cancer: evidence for three tumour-suppressor loci. Br J Cancer 73: 420-423, 1996.

30. Lu KH, Weitzel JN, Kodali S, Welch WR, Berkowitz RS and Mok SC: A novel 4-cM minimally deleted region on chromosome $11 \mathrm{p} 15.1$ associated with high grade nonmucinous epithelial ovarian carcinomas. Cancer Res 57: 387-390, 1997.

31. Wertheim I, Tangir J, Muto MG, Welch WR, Berkowitz RS, Chen WY, et al: Loss of heterozygosity of chromosome 17 in human borderline and invasive epithelial ovarian tumors. Oncogene 12: 2147-2153, 1996.

32. Edelson MI, Lau CC, Colitti CV, Welch WR, Bell DA, Berkowitz RS, et al: A one centimorgan deletion unit on chromosome Xq12 is commonly lost in borderline and invasive epithelial ovarian tumors. Oncogene 16: 197-202, 1998.

33. Herman JG, Umar A, Polyak K, Graff JR, Ahuja N, Issa JP, et al: Incidence and functional consequences of hMLH1 promoter hypermethylation in colorectal carcinoma. Proc Natl Acad Sci USA 95: 6870-6875, 1998.

34. Georgiades IB, Curtis LJ, Morris RM, Bird CC and Wyllie AH: Heterogeneity studies identify a subset of sporadic colorectal cancers without evidence for chromosomal or microsatellite instability. Oncogene 18: 7933-7940, 1999.

35. Jallepalli PV, Waizenegger IC, Bunz F, Langer S, Speicher MR, Peters JM, et al: Securin is required for chromosomal stability in human cells. Cell 105: 445-457, 2001.

36. Nathke IS: The adenomatous polyposis coli protein. Mol Pathol 52: 169-173, 1999.

37. Toyota M, Ho C, Ahuja N, Jair KW, Li Q, Ohe-Toyota M, et al: Identification of differentially methylated sequences in colorectal cancer by methylated $\mathrm{CpG}$ island amplification. Cancer Res 59: 2307-2312, 1999

38. Ahuja N, Li Q, Mohan AL, Baylin SB and Issa JP: Aging and DNA methylation in colorectal mucosa and cancer. Cancer Res 58: 5489-5494, 1998.

39. Esteller M, Corn PG, Baylin SB and Herman JG: A gene hypermethylation profile of human cancer. Cancer Res 61: 3225-3229, 2001

40. Lynch HT, Smyrk T and Lynch JF: Overview of natural history, pathology, molecular genetics and management of HNPCC (Lynch Syndrome). Int J Cancer 69: 38-43, 1996.

41. Kavanagh J, Tresukosol D, Edwards C, Freedman R, Gonzalez de Leon C, Fishman A, et al: Carboplatin reinduction after taxane in patients with platinum-refractory epithelial ovarian cancer. J Clin Oncol 13: 1584-1588, 1995.

42. Anthoney DA, McIlwrath AJ, Gallagher WM, Edlin AR and Brown R: Microsatellite instability, apoptosis, and loss of p53 function in drug-resistant tumor cells. Cancer Res 56: 1374-1381, 1996.

43. Gupta PK, Sahota A, Boyadjiev SA, Bye S, Shao C, O'Neill JP, et al: High frequency in vivo loss of heterozygosity is primarily a consequence of mitotic recombination. Cancer Res 57: 1188-1193, 1997.

44. Cahill DP, Kinzler KW, Vogelstein B and Lengauer C: Genetic instability and darwinian selection in tumours. Trends Cell Biol 9: M57-M60, 1999. 